\title{
Contact orderability up to conjugation
}

\author{
Kai Cieliebak ${ }^{1 *} \quad$ Yakov Eliashberg ${ }^{2 \dagger} \quad$ Leonid Polterovich ${ }^{3 \ddagger}$ \\ ${ }^{1}$ Institut für Mathematik, Universität Augsburg, Germany \\ ${ }^{2}$ Department of Mathematics, Stanford University, USA \\ ${ }^{3}$ Department of Mathematics, Tel Aviv University, Israel
}

September 28, 2017

To the memory of Vladimir Igorevich Arnold

\section{Contents}

1 Introduction 2

1.1 Groups of contactomorphisms and their Lie algebras . . . . . 2

1.2 Dominating positive cones . . . . . . . . . . . . 3

1.3 Contact manifolds convex at infinity . . . . . . . . . . . 4

1.4 The maximal dominating cone $\mathfrak{g}^{+} \ldots \ldots$. . . . . . . 6

1.5 Partial order on $\mathfrak{g}^{+}$up to conjugation . . . . . . . . 8

2 Orderability and symplectic non-squeezing 10

2.1 The class $\mathfrak{C}_{k}$ of unbounded domains in $\mathbb{R}^{2 n} \ldots \ldots \ldots$

2.2 Capacity-like function on $\mathfrak{C}_{k}$ and proof of Theorem 1.11. . . . 14

3 Invariants of domains from $\mathfrak{C}_{k}$

3.1 Floer-Hofer symplectic homology of bounded domains in $\mathbb{R}^{2 n}$. 15

3.2 Symplectic homology for domains from $\mathfrak{C}_{k} \ldots \ldots \ldots$

*Partially supported by DFG grant CI 45/8-1

${ }^{\dagger}$ Partially supported by NSF grant DMS-1505910

$\ddagger$ Partially supported by the ISF grant 178/13 and the ERC Advanced grant 338809 
3.3 A capacity for domains from $\mathfrak{C}_{k} \ldots \ldots \ldots$

3.4 Proof of Theorem $2.3 \ldots \ldots \ldots$

3.5 Proof of Proposition $3.2 \ldots \ldots \ldots$

4 Geometry of the dominating positive cone 25

\section{Introduction}

A partial order on groups of contact diffeomorphisms was introduced in [13] as a contact analog of Hofer's geometry for groups of Hamiltonian diffeomorphisms of symplectic manifolds. In this paper we begin studying the remnants of this order on the conjugacy classes of contactomorphisms. Our main interest in this paper are non-compact contact manifolds, and more specifically a special class of non-compact contact manifolds which we call convex at infinity, see Section 1.3 below. While orderability problems for closed manifolds have obvious answers on the level of Lie algebra of contact vector fields, the situation for non-compact manifolds is quite subtle already on the Lie algebra level. Problems of this kind naturally arisen in connection with constructions of contact structures in [1]. The goal of the paper is to illustrate the arising phenomena on a restricted class of examples, leaving a more general study, both in the Lie algebra and the group cases, to our forthcoming paper [5].

\subsection{Groups of contactomorphisms and their Lie alge- bras}

Let $(U, \xi)$ be a coorientable noncompact contact manifold. We fix a contact form $\alpha$ for $\xi$ and denote by $R$ its Reeb vector field. Let

$$
G:=\operatorname{Diff}_{c}(U, \xi)
$$

be the identity component of the group of contactomorphisms of $(U, \xi)$ with compact support. The Lie algebra $\mathfrak{g}$ of $G$, which consists of compactly supported contact vector fields, can be identified with the space $C_{c}^{\infty}(U)$ of smooth functions with compact support by associating to each function $K$ its contact vector field

$$
Y_{K}=K R+Z_{K}, \quad Z_{K} \in \xi,\left.\quad\left(d K+i_{Z_{K}} d \alpha\right)\right|_{\xi}=0
$$


Note that

$$
d K\left(Z_{K}\right)=0, \quad L_{Y_{K}} \alpha=d K(R) \alpha .
$$

Conversely, given a contact vector field $Y$ its contact Hamiltonian is defined by the formula $K(x)=\alpha(Y(x)), x \in U$. Let us stress the point that to identify the Lie algebra $\mathfrak{g}$ with the function space $C_{c}^{\infty}(U)$ one needs to fix a contact form.

The adjoint action of $\psi \in G$ on $K \in \mathfrak{g}$ computes to

$$
\operatorname{Ad}_{\psi} K=\left(c_{\psi} K\right) \circ \psi^{-1}
$$

where $c_{\psi}: U \rightarrow \mathbb{R}$ is the positive function satisfying $\psi^{*} \alpha=c_{\psi} \alpha$. The Lie bracket on $\mathfrak{g}$ is given by

$$
\{H, K\}=d K\left(X_{H}\right)-K d H(R) \text {. }
$$

The Lie algebra carries a canonical partial order defined by $H \leq K$ if $H(x) \leq$ $K(x)$ for all $x \in M$, which is Ad-invariant by equation (1).

\subsection{Dominating positive cones}

Denote by $\mathfrak{g}^{\geq 0}$ the cone in the Lie algebra $\mathfrak{g} \cong C_{c}^{\infty}(U)$ consisting of nonnegative functions.

Definition 1. A subcone $\mathfrak{c} \subset \mathfrak{g}^{\geq 0} \backslash 0$ is called a dominating (positive) cone if the following hold:

(i) $\mathfrak{c}$ is Ad-invariant;

(ii) $\mathfrak{c}$ is relatively open in $\mathfrak{g}^{\geq 0} \backslash\{0\}$;

(iii) for each $H \in \mathfrak{g}$ there exists $K \in \mathfrak{c}$ with $H \leq K$;

(iv) for all $H \in \mathfrak{g}, K \in \mathfrak{c}$ there exist $t>0$ and $g \in G$ such that $t \operatorname{Ad}_{g} H \leq K$;

(v) for each $H \in \mathfrak{g}^{\geq 0} \backslash\{0\}$ there exist $g_{1}, \ldots, g_{k} \in G$ such that $\operatorname{Ad}_{g_{1}} H+$ $\cdots+\operatorname{Ad}_{g_{k}} H \in \mathfrak{c}$.

Remark 1.1. Property (v) is not needed in this paper, but will become relevant for the discussion of partial orders on contactomorphism groups in [5].

Clearly, if the manifold $U$ is closed then the only dominating cone in $\mathfrak{g}$ is the cone $\mathfrak{g}^{>0}$ consisting of everywhere positive functions. If $U$ is not closed, then a dominating cone in general need not exist. For instance, $S^{1} \times \mathbb{R}^{2}$ with the 
contact form $d t+\frac{1}{2}(x d y-y d x)$ does not admit any dominating cone because if $\operatorname{supp}(H) \supset S^{1} \times D_{R}$ and $\operatorname{supp}(K) \subset S^{1} \times D_{r}$ with $r<R$, then there is no contactomorphism $g \subset G$ such that $g(\operatorname{supp}(H)) \subset \operatorname{supp}(K)$, see [8].

However, there is an important class of noncompact contact manifolds, called convex at infinity, for which a dominating cone always exists. We discuss this class in the next subsection.

\subsection{Contact manifolds convex at infinity}

A noncompact contact manifold $(U, \xi)$ is called convex at infinity if there exists a contact embedding $\sigma: U \hookrightarrow U$ which is contactly isotopic to the identity such that $\sigma(U) \Subset U$, i.e $\sigma(U)$ has a compact closure in $U$. The space of all embeddings $\sigma$ with this property will be denoted by

$$
\mathcal{E}=\mathcal{E}(U, \xi) .
$$

Note that by cutting off a contact isotopy, the restriction $\left.\sigma\right|_{K}$ to any compact set $K \subset U$ can be extended to a contactomorphism in the group $G=\operatorname{Diff}_{c}(U, \xi)$.

The notion of contact convexity for hypersurfaces in a contact manifolds was introduced in [11] and studied in detail in [16]. Let us recall that a hypersurface in a contact manifold is called convex if it admits a transverse contact vector field. The coorientation of this vector field is irrelevant because if $Y$ is contact then $-Y$ is contact as well.

Example 1.2. (i) A major class of contact manifolds convex at infinity is provided by interiors of compact manifolds with convex boundary. Indeed, as the required embedding $\sigma$ one can take the flow for small positive time of an inward pointing contact vector field transverse to the boundary.

(ii) More generally, suppose a contact manifold $(U, \xi)$ admits a (not necessarily complete) contact vector field $Y$ without zeroes at infinity, which outside a compact set is gradient-like for an exhausting function $\phi: U \rightarrow \mathbb{R}$. Then $(U, \xi)$ is convex at infinity. Indeed, first use [10, Lemma 2.6] to conclude that for a sufficiently large $c$ the end $(\{\phi \geq c\}, \xi)$ is contactomorphic to $(\Sigma \times[0, \infty), \widehat{\xi})$ such that the vector field $\frac{\partial}{\partial s}$ is contact. Here we set $\Sigma:=\{\phi=c\}$ and denoted by $s$ the coordinate corresponding to the second factor. There is a contact isotopy $h_{t}:(\Sigma \times[0, \infty), \widehat{\xi}) \rightarrow(\Sigma \times[0, \infty), \widehat{\xi})$, $t \in[0,1]$, such that that $h_{0}=\mathrm{id}, h_{t}=\mathrm{id}$ near $\Sigma \times 0$ for all $t \in[0,1]$ and $h_{1}(\Sigma \times n)=\Sigma \times \frac{n-1}{n}, n=1, \ldots$, which implies that $h_{1}(\Sigma \times[0, \infty))=\Sigma \times[0,1)$.

(iii) In a 3-dimensional contact manifold a generic surface is convex, see [16], hence the interior of a generic connected compact contact manifold with 
non-empty boundary is convex at infinity. If the boundary components of a 3-manifold are 2-spheres and the manifold is tight near the boundary, then it is convex at infinity, even when the boundary components are not convex, see [9].

(iv) On the other hand, the contact manifold $S^{1} \times \mathbb{R}^{2}=(\mathbb{R} / \mathbb{Z}) \times \mathbb{R}^{2}$ with the tight contact form $d t+\frac{1}{2}(x d y-y d x)$ is not convex at infinity, see [8].

Now we will introduce our main example. Let $\lambda_{\mathrm{st}}=\frac{1}{2} \sum_{1}^{n}\left(x_{i} d y_{i}-y_{i} d x_{i}\right)$ be the standard Liouville form on $\mathbb{R}^{2 n}$ with its Liouville vector field

$$
Z=\frac{1}{2} \sum_{1}^{n}\left(x_{i} \frac{\partial}{\partial x_{i}}+y_{i} \frac{\partial}{\partial y_{i}}\right),
$$

and let $\alpha_{\text {st }}=\left.\lambda_{\text {st }}\right|_{S^{2 n-1}}$ be the standard contact form on the unit sphere $S^{2 n-1} \subset \mathbb{R}^{2 n}$. Let us order coordinates in $\mathbb{R}^{2 n}$ as $\left(x_{1}, \ldots, x_{n}, y_{1}, \ldots, y_{n}\right)$ and denote by $\Pi_{k}$ a $k$-dimensional coordinate subspace of $\mathbb{R}^{2 n}$ which is spanned by the last $k$ vectors of the basis. For instance, $\Pi_{1}$ is the $y_{n}$-coordinate axis, while $\Pi_{2 n-1}$ is the hyperplane $\left\{x_{1}=0\right\}$. Note that $\Pi_{k}$ is isotropic when $k \leq n$, and coisotropic otherwise. We denote by $\Pi_{k}^{\perp}$ the orthogonal subspace spanned by the first $2 n-k$ basic vectors.

Lemma 1.3. (a) For each $k=1, \ldots, 2 n-1$ the contact manifold $\left(S^{2 n-1}\right)$ $\left.\Pi_{k}, \xi_{\mathrm{st}}\right)$ is convex at infinity. Moreover, it can be contracted by an element of $\mathcal{E}$ to an arbitrarily small neighborhood of the equatorial sphere $S^{2 n-1} \cap \Pi_{k}^{\perp}$.

(b) For $k \geq n$ the manifold $\left(S^{2 n-1} \backslash \Pi_{k}, \xi_{\text {st }}\right)$ is contactomorphic to $J^{1}\left(S^{2 n-k-1}\right) \times$ $\mathbb{R}^{2 k-2 n}=T^{*}\left(S^{2 n-k-1} \times \mathbb{R}^{k-n}\right) \times \mathbb{R}$.

Proof. Recall that $r=|x|^{2}+|y|^{2}$ induces the canonical isomorphism $\left(\mathbb{R}^{2 n} \backslash\right.$ $\left.\{0\}, \lambda_{\text {st }}\right) \cong\left(\mathbb{R}_{+} \times S^{2 n-1}, r \alpha_{\text {st }}\right)$ under which the Liouville vector field $Z$ corresponds to $r \frac{\partial}{\partial r}$. Thus contact vector fields on $S^{2 n-1}$ are in one-to-one correspondence with Hamiltonian vector fields on $\mathbb{R}^{2 n} \backslash\{0\}$ which commute with $Z$. Note that each linear vector field on $\mathbb{R}^{2 n}$ automatically commutes with $Z$.

(a) First consider the case $k \leq n$. The linear vector field

$$
\widehat{Y}_{k}:=\sum_{j=n-k+1}^{n}\left(-x_{j} \frac{\partial}{\partial x_{j}}+y_{j} \frac{\partial}{\partial y_{j}}\right)
$$

is the Hamiltonian vector field of the function $\sum_{n-k+1}^{n} x_{j} y_{j}$. It commutes with $Z$, so it descends to a contact vector field $Y_{k}$ to $S^{2 n-1}$. On $\mathbb{R}^{2 n} \backslash\left(\Pi_{k} \cup \Pi_{k}^{\perp}\right)$ the 
field $\widehat{Y}_{k}$ is gradient-like for the $Z$-invariant function $-\ln \left(1-\frac{\sum_{n-k+1}^{n} y_{j}^{2}}{|x|^{2}+|y|^{2}}\right)$, hence on $S^{2 n-1} \backslash\left(\Pi_{k} \cup \Pi_{k}^{\perp}\right)$ the field $Y_{k}$ has no zeroes and is gradient-like for the exhausting function $-\ln \left(1-\sum_{n-m+1}^{n} y_{j}^{2}\right)$. Now convexity at infinity follows from Example 1.2(ii), and the flow of $Y_{k}$ for very negative times contracts any compact set $S^{2 n-1} \backslash \Pi_{k}$ to a neighborhood of $S^{2 n-1} \cap \Pi_{k}^{\perp}$.

The case $k>n$ follows from part (b) and Example 1.2(ii).

(b) For $k \geq n$ let

$$
\widehat{Z}_{k}:=\sum_{j=1}^{2 n-k}\left(x_{j} \frac{\partial}{\partial x_{j}}-y_{j} \frac{\partial}{\partial y_{j}}\right)
$$

be the Hamiltonian vector field of the function $-\sum_{1}^{2 n-k} x_{j} y_{j}$. It descends to a complete contact vector field $Z_{k}$ on $S^{2 n-1}$. The flow of $Z_{k}$ contracts every compact set in $S^{2 n-1} \backslash \Pi_{k}$ to a neighborhood of the isotropic sphere $S^{2 k-1} \cap \Pi_{k}^{\perp}$ and the field $-Z_{k}$ is gradient like for the function $\left(\sum_{1}^{n} y_{j}^{2}+\sum_{2 n-k+1}^{n} x_{j}^{2}\right)$ on $S^{2 n-1} \backslash \Pi_{k}$. By Weinstein-Darboux theorem contact structures on $S^{2 n-1} \backslash \Pi_{k}$ and $J^{1}\left(S^{2 n-k-1}\right) \times \mathbb{R}^{k-n}$ are isomorphic on tubular neighborhoods of isotropic sphere $S^{2 n-k-1}=S^{2 n-1} \cap \Pi_{k}^{\perp}$ and the 0-section $S^{2 n-k-1} \times 0 \subset J^{1}\left(S^{2 n-k-1}\right) \times$ $\mathbb{R}^{2 k-2 n}$. This isomorphism then extends to a contactomorphism between $S^{2 n-1} \backslash \Pi_{k}$ and $J^{1}\left(S^{2 n-k-1}\right) \times \mathbb{R}^{2 k-2 n}$ by matching the corresponding trajectories of the contact vector field $-Z_{k}$ with trajectories of the canonical contact vector field on $J^{1}\left(S^{2 n-k-1}\right) \times \mathbb{R}^{2 k-2 n}$ contracting this manifold to its 0 -section.

\subsection{The maximal dominating cone $\mathfrak{g}^{+}$}

Let $(U, \xi)$ be a contact manifold convex at infinity.

Lemma 1.4. For $(U, \xi)$ connected and convex at infinity the cone

$$
\mathfrak{g}^{+}:=\left\{H \in \mathfrak{g}^{\geq 0}|H|_{\sigma(U)}>0 \text { for some } \sigma \in \mathcal{E}(U, \xi)\right\} .
$$

is dominating and maximal, (i.e., all other dominating cones are subcones of $\left.\mathfrak{g}^{+}\right)$.

Proof. Properties (i), (ii) and (iii) in Definition 1 are clear.

For (iv), consider $H \in \mathfrak{g}, K \in \mathfrak{g}^{+}$. Then $C:=\operatorname{supp}(H)$ is compact and $K$ is positive on $\sigma(U)$ for some $\sigma \in \mathcal{E}(U, \xi)$. By cutting off the contact isotopy 
from the identity to $\sigma$ outside $C$ we find $g \in G$ with $\left.g\right|_{C}=\left.\sigma\right|_{C}$. Then $\operatorname{supp}\left(\operatorname{Ad}_{g} H\right)=g(\operatorname{supp}(H))=\sigma(C) \subset \sigma(U)$. Since $\left.K\right|_{\sigma(U)}>0$, it follows that $t \operatorname{Ad}_{g} H \leq K$ for $t$ sufficiently small.

For (v), let $H \in \mathfrak{g}^{\geq 0} \backslash\{0\}$ be strictly positive on some open set $V \subset U$. Pick any $\sigma \in \mathcal{E}(U, \xi)$. Since the group $G$ acts transitively on $U$ and $\sigma(U)$ is relatively compact, there exist $g_{1}, \ldots, g_{k} \in G$ such that $\sigma(U) \subset g_{1}(V) \cup$ $\cdots \cup g_{k}(V)$. Then $\operatorname{Ad}_{g_{i}} H$ is nonnegative and strictly positive on $g_{i}(V)$, hence $\operatorname{Ad}_{g_{1}} H+\cdots+\operatorname{Ad}_{g_{k}} H$ is strictly positive on $\sigma(U)$ and therefore belongs to $\mathfrak{g}^{+}$.

To prove maximality of $\mathfrak{g}^{+}$, let $\mathfrak{c}$ be any other dominating cone. Take any $H \in \mathfrak{c}$ and $F \in \mathfrak{g}^{+}$. Then by Definition 1 there exists $K \in \mathfrak{c}$ such that $F \leq K$, and there exist $g \in G$ and $t>0$ such that $t \operatorname{Ad}_{g} K \leq H$. It follows that $t \operatorname{Ad}_{g} F \leq H$. Since $F \in \mathfrak{g}^{+}$, this implies that $H$ is positive on $\sigma(U)$ for some $\sigma \in \mathcal{E}$, and therefore $H \in \mathfrak{g}^{+}$.

Lemma 1.5 (Examples of maximal dominating cones).

(i) For the standard contact structure on $\mathbb{R}^{2 n+1}$ we have $\mathfrak{g}^{+}=\mathfrak{g}^{\geq 0} \backslash 0$.

(ii) For the 1-jet space $U=J^{1}(M)$ of a closed manifold $M$ endowed with its standard contact structure, the maximal dominating cone $\mathfrak{g}^{+}$consists of all nonnegative functions whose support contains a neighborhood of a Legendrian submanifold isotopic to the zero section.

(iii) For $\left(S^{2 n-1} \backslash \Pi_{k}, \xi_{\mathrm{st}}\right)$ as in Example (iii) in Section 1.3, the cone $\mathfrak{g}^{+}$ consists of all nonnegative functions which are positive on an image of the equatorial sphere $S^{2 n-1} \cap \Pi_{k}^{\perp}$ under a contactomorphism isotopic to the identity.

(iv) In the special case $\left(S^{3} \backslash \Pi_{1}, \xi_{\mathrm{st}}\right)$, which is the same as $\mathbb{R}^{3} \backslash 0$ with the standard contact structure inherited from $\mathbb{R}^{3}$, the cone $\mathfrak{g}^{+}$can also be characterized as consisting of all nonnegative functions whose support contains a neighborhood homologically non-trivial 2-sphere.

Proof. Both contact manifolds in (i) and (ii) admit complete contact vector fields which contract every compact subset to an arbitrarily small neighborhood of the origin in case (i), and to an arbitrarily small neighborhood of the zero section in case (ii). For $\left(\mathbb{R}^{2 n-1}, d t+\sum_{1}^{n-1}\left(x_{j} d y_{j}-y_{j} d x_{j}\right)\right)$ this is the vector field $-2 \frac{\partial}{\partial t}-\sum_{1}^{n-1}\left(x_{j} \frac{\partial}{\partial x_{j}}+y_{j} \frac{\partial}{\partial y_{j}}\right)$, and for $\left(J^{1}(M), d z+p d q\right)$ this is the vector field $-\frac{\partial}{\partial z}-p \frac{\partial}{\partial p}$. But the group $G$ acts transitively on points and on Legendrian submanifolds isotopic to the zero section, respectively. In (iii), 
according to Lemma 1.3 the space $S^{2 n-1} \backslash \Pi_{k}$ can be contracted by an element in $\mathcal{E}$ to a neighborhood of the equatorial sphere $S^{2 n-1} \cap \Pi_{k}^{\perp}$. For (iv), we note in addition that any two smoothly isotopic 2 -spheres in a tight contact manifold can be $C^{0}$-approximated by spheres which are contactly isotopic, see [9].

Remark 1.6. If $U$ contains a compact subset which is not contractible in $U$, then the cone $\mathfrak{g}^{+}$never coincides with $\mathfrak{g}^{\geq 0} \backslash\{0\}$. To see this, pick $H, K \in \mathfrak{g}^{\geq 0} \backslash$ $\{0\}$ such that $\operatorname{supp}(H)$ is noncontractible in $U$ and $\operatorname{supp}(K)$ is contractible in $U$. Suppose there exists $g \in G$ and $t>0$ with $t \operatorname{Ad}_{g} H \leq K$. Then we must have $g(\operatorname{supp}(H)) \subset \operatorname{supp}(K)$, which is impossible if $\operatorname{supp}(H)$ is noncontractible and $\operatorname{supp}(K)$ is contractible in $U$.

\subsection{Partial order on $\mathfrak{g}^{+}$up to conjugation}

Let us denote by $\Theta:=\mathfrak{g}^{+} / \sim$ the quotient space of $\mathfrak{g}^{+}$by the adjoint action of $G$ on $\mathfrak{g}$. The partial order $H \leq K$ on $\mathfrak{g}^{+}$descends to a possibly degenerate partial order $\preceq$ on $\Theta$ defined on $h, k \in \Theta$ by

$$
h \preceq k: \Longleftrightarrow \text { there exists } H \in h, K \in k \text { such that } H \leq K .
$$

Lemma 1.7. The following are equivalent:

(a) there exists $H \in \mathfrak{g}^{+}$and $g \in G$ such that $\operatorname{Ad}_{g} H \leq s H$ for some $0<s<1$;

(b) for all $K_{1}, K_{2} \in \mathfrak{g}^{+}$there exists $h \in G$ such that $\operatorname{Ad}_{h} K_{1} \leq K_{2}$.

Proof. Clearly (b) implies (a). Conversely, suppose that (a) holds for elements $H, g$ and let $K_{1}, K_{2} \in \mathfrak{g}^{+}$be given. By Definition 1 there exist $t_{i}>0$ and $h_{i} \in G$ such that

$$
t_{1} \operatorname{Ad}_{h_{1}} K_{1} \leq H \leq \frac{1}{t_{2}} \operatorname{Ad}_{h_{2}} K_{2} .
$$

Applying $\operatorname{Ad}_{g}^{N}$ for some $N \in \mathbb{N}$ to these inequalities, we obtain

$$
t_{1} \operatorname{Ad}_{g}^{N} \operatorname{Ad}_{h_{1}} K_{1} \leq \operatorname{Ad}_{g}^{N} H \leq s^{N} H \leq \frac{s^{N}}{t_{2}} \operatorname{Ad}_{h_{2}} K_{2} .
$$

Applying $\operatorname{Ad}_{h_{2}}^{-1}$ to both sides and dividing by $t_{1}$, we obtain

$$
\operatorname{Ad}_{h_{2}}^{-1} \operatorname{Ad}_{g}^{N} \operatorname{Ad}_{h_{1}} K_{1} \leq \frac{s^{N}}{t_{1} t_{2}} K_{2} .
$$

Hence $\operatorname{Ad}_{h} K_{1} \leq K_{2}$ with $h:=h_{2}^{-1} g^{N} h_{1}$, provided that $N$ is chosen so large that $s^{N} \leq t_{1} t_{2}$. 
We call the positive cone $\mathfrak{g}^{+}$non-orderable up to conjugation if the equivalent conditions in Lemma 1.7 hold, and orderable up to conjugation otherwise. Thus to prove orderability up to conjugation of $\mathfrak{g}^{+}$, it suffices to find some pair $K_{1}, K_{2} \in \mathfrak{g}^{+}$for which there exists no $h \in G$ with $\operatorname{Ad}_{h} K_{1} \leq K_{2}$.

Remark 1.8. a) Even if $\mathfrak{g}^{+}$is orderable up to conjugation this does not imply that the induced binary relation on $\Theta$ is a genuine order. However, we do not know any counterexamples to this implication. We will discuss the arising structures in more detail in Section 4 below.

b) If the manifold $U$ is closed then the cone $\mathfrak{g}^{+}$is always orderable up to conjugation for the following trivial reason: the volume integral

$$
I(H):=\int_{U}\left(\frac{\alpha}{H}\right) \wedge d\left(\frac{\alpha}{H}\right)^{n-1}
$$

satisfies $I\left(\operatorname{Ad}_{g} H\right)=I(H)$ for all $g \in G$, so one can never have $\operatorname{Ad}_{g} H \leq s H$ for some $0<s<1$. Note that the strict order $H>G$ does descend in the case of a closed $U$ to a genuine order on $\Theta$, as it follows from the same preservation of volume argument.

Proposition 1.9. (a) If $(U, \xi)$ is the standard contact $\mathbb{R}^{2 n+1}$ or $J^{1}(M)$, as in Lemma 1.5 (i) and (ii), then $\mathfrak{g}^{+}$is non-orderable up to conjugation.

(b) More generally, let $(V, \lambda)$ be the completion of a Liouville domain (see [4]). Then for its contactization $(U=V \times \mathbb{R}, \operatorname{ker}(\lambda+d t))$ the maximal dominating cone $\mathfrak{g}^{+}(U, \xi)$ is non-orderable up to conjugation.

Proof. Since (a) follows from (b), it suffices to prove (b). The Liouville flow $\phi_{s}$ on $V$ induces a contact diffeotopy $\psi_{s}(x, t)=\left(\phi_{s}(x), e^{s} t\right)$ of $V \times \mathbb{R}$ satisfying $\psi_{s}^{*}(\lambda+d t)=e^{s}(\lambda+d t)$. Let $C \subset V \times \mathbb{R}$ be the attractor of the flow $\psi_{s}$ when $s \rightarrow-\infty$. Take $K_{1}, K_{2} \in \mathfrak{g}^{+}, K_{1} \geq K_{2}$. By the definition of the cone $\mathfrak{g}^{+}$there exists a contacomorphism $h \in G$ such that $\operatorname{supp}\left(\operatorname{Ad}_{h} K_{2}\right)$ contains a neighborhood of $C$. The flow $\psi_{s}$ when $s \rightarrow-\infty$ moves $\operatorname{supp}\left(K_{1}\right)$ into an arbitrarily small neighborhood of $C$, and hence for sufficiently large $-s$ we have $\operatorname{supp}\left(\operatorname{Ad}_{\psi_{s}}\left(K_{1}\right)=\operatorname{supp}\left(K_{1} \circ \psi_{s}^{-1}\right) \subset \operatorname{supp}\left(\operatorname{Ad}_{h} K_{2}\right)\right.$. Therefore, $\left(\operatorname{Ad}_{\psi_{s}} \widetilde{K}_{1}\right)(x)=e^{s} K_{1}\left(\psi_{s}^{-1}(x)\right) \leq \operatorname{Ad}_{h} K_{2}(x)$ for for sufficiently large $-s$, or $\left(\operatorname{Ad}_{h^{-1} \circ \psi_{s}} K_{1}\right)(x) \leq K_{2}(x)$, which means that $\mathfrak{g}^{+}$satisfies condition (b) in Lemma 1.7.

Proposition 1.9(b) combined with Lemma 1.3(b) yields

Corollary 1.10. If $k \geq n$, then for $\left(S^{2 n-1} \backslash \Pi_{k}, \xi_{\mathrm{st}}\right)$ from Section 1.3 the cone $\mathfrak{g}^{+}$is non-orderable up to conjugation. 
By contrast, we will show below in Section 2.2

Theorem 1.11. If $k<n$, then for $\left(S^{2 n-1} \backslash \Pi_{k}, \xi_{\mathrm{st}}\right)$ the cone $\mathfrak{g}^{+}$is orderable up to conjugation: there exist $H, K \in \mathfrak{g}^{+}$for which there is no $g \in G$ with $\operatorname{Ad}_{g} H \leq K$. More precisely, there exists a surjective map $w: \mathfrak{g}^{+} \rightarrow(0, \infty)$ such that $w\left(\operatorname{Ad}_{g} H\right)=w(H)$ for $g \in G, w(s H)=s^{-2} w(H)$ for any $s>0$ and such that $H \leq K$ implies $w(H) \geq w(K)$.

\section{Orderability and symplectic non-squeezing}

In this section we will rephrase Theorem 1.11 as a non-squeezing result for suitable unbounded domains in the standard symplectic space $\left(\mathbb{R}^{2 n}, \omega=\right.$ $\left.\sum_{1}^{n} d x_{j} \wedge d y_{j}\right)$. Throughout this section we fix $k$ with $1 \leq k \leq n$ and denote

$$
U_{k}:=S^{2 n-1} \backslash \Pi_{k}, \quad G_{k}:=\operatorname{Diff}_{c}\left(U_{k}, \xi_{\mathrm{st}}\right) \text {. }
$$

\subsection{The class $\mathfrak{C}_{k}$ of unbounded domains in $\mathbb{R}^{2 n}$}

Introduce "polar coordinates", $r=|x|^{2}+|y|^{2} \in \mathbb{R}, \theta=r^{-1 / 2}(x, y) \in S^{2 n-1}$, so that the standard Liouville form $\lambda_{\text {st }}$ can be written as

$$
\lambda_{\mathrm{st}}=\frac{1}{2} \sum_{1}^{n}\left(x_{j} d y_{j}-y_{j} d x_{j}\right)=r \alpha_{\mathrm{st}},
$$

where $\alpha_{\mathrm{st}}$ is the standard contact form on the unit sphere $S^{2 n-1}$ which defines the standard contact structure $\xi_{\mathrm{st}}=\operatorname{ker} \alpha_{\mathrm{st}}$. The coordinates $(r, \theta)$ identify $\left(\mathbb{R}^{2 n} \backslash 0, \lambda_{\text {st }}\right)$ with the symplectization $\left(\mathbb{R}_{+} \times S^{2 n-1}, r \alpha_{\mathrm{st}}\right)$ of the standard contact structure on $S^{2 n-1}$. Thus the symplectization of $U_{k}=S^{2 n-1} \backslash \Pi_{k}$ gets identified with $\mathbb{R}^{2 n} \backslash \Pi_{k}$.

Note that any contactomorphism $\phi$ of $\left(S^{2 n-1}, \xi_{\text {st }}\right)$ defines a symplectomorphism $S \phi: \mathbb{R}^{2 n} \rightarrow \mathbb{R}^{2 n}$, singular at the origin, by the formula

$$
S \phi(r, \theta):=\left(\frac{r}{c_{\phi}(\theta)}, \phi(\theta)\right)
$$

where $\phi^{*} \alpha_{\text {st }}=c_{\phi}(\theta) \alpha_{\text {st }}$.

Lemma 2.1. If $\phi$ is contactly isotopic to the identity, then there exists a constant $K_{\phi}>1$ such that for any $\varepsilon>0$ there exists a smooth symplectomorphism $S_{\varepsilon} \phi$ of $\mathbb{R}^{2 n}$ which equals the identity on the $\varepsilon$-ball around the origin and which coincides with $S \phi$ outside the $\left(K_{\phi} \varepsilon\right)$-ball. Moreover, if $\phi$ as well as its isotopy to the identity equal the identity near some compact subset $C \subset S^{2 n-1}$, then $S_{\varepsilon} \phi$ can be chosen equal to the identity on the cone over $C$. 
Proof. Let $\phi_{t}, t \in[0,1]$ be a contact isotopy of $\left(S^{2 n-1}, \xi_{\mathrm{st}}\right)$ connecting $\phi_{0}=\mathrm{id}$ to $\phi_{1}=\phi$ with $\phi_{i}=$ id on $C$. Let $S \phi_{t}: \mathbb{C}^{n} \backslash 0 \rightarrow \mathbb{C}^{n} \backslash 0$ be its symplectization. Set

$$
M:=\max _{t \in[0,1]} \max \left(c_{\phi_{t}}\right), \quad m:=\min _{t \in[0,1]} \min \left(c_{\phi_{t}}\right)
$$

and note that $m \leq 1 \leq M$. Then for any $\delta>0, t \in[0,1]$ we have

$$
\left\{r \leq \frac{\delta}{M}\right\} \subset S \phi_{t}(\{r \leq \delta\}) \subset\left\{r \leq \frac{\delta}{m}\right\} .
$$

Therefore, we can extend the Hamiltonian isotopy $\left.S \phi_{t}\right|_{\{r \geq \delta\}}$ to a Hamiltonian isotopy $g_{t}: \mathbb{C}^{n} \rightarrow \mathbb{C}^{n}$ such that $g_{0}=\mathrm{id}$ and for all $t \in[0,1]$

$$
\left.g_{t}\right|_{\left\{r \leq \frac{\delta}{2 M}\right\} \cup\{\theta \in K\}}=\mathrm{id},\left.\quad g_{t}\right|_{\left\{r \geq \frac{2 \delta}{m}\right\}}=S \phi_{t} .
$$

Then for $\delta=2 M \varepsilon$ the symplectomorphism $S_{\varepsilon} \phi:=g_{1}$ satisfies the required conditions with $K_{\phi}=\frac{4 M}{m}$.

Given a nonnegative compactly supported contact Hamiltonian $H: U_{k} \rightarrow \mathbb{R}$ we extend it by 0 to $S^{2 n-1}$ and will keep the notation $H$ for this extension. Recall that according to Lemma 1.5 the cone $\mathfrak{g}^{+}=\mathfrak{g}^{+}\left(U_{k}\right)$ consists of all functions satisfying the conditions

(i) $H=0$ near $S^{2 n-1} \cap \Pi_{k}=S^{2 n-1} \cap\left\{x=0, y_{1}=\cdots=y_{n-k}=0\right\}$;

(ii) $H$ is positive on the image $g\left(S^{2 n-1} \cap \Pi_{k}^{\perp}\right)$ of the equator under a contactomorphism $g \in G_{k}$.

We now define a class $\mathfrak{C}_{k}$ of domains in $\mathbb{R}^{2 n}$ which, in particular (see Lemma 2.2 below), contains all the domains of the form

$$
V(H):=\left\{(r, \theta) \in \mathbb{R}_{+} \times S^{2 n-1} \mid r H(\theta)<1\right\}, \quad H \in \mathfrak{g}^{+}\left(U_{k}\right) .
$$

First, we add to $\mathfrak{C}_{k}$ all hyperboloids

$$
V_{k}^{a, b}:=\left\{\frac{1}{a^{2}}\left(\sum_{1}^{n} x_{i}^{2}+\sum_{1}^{n-k} y_{i}^{2}\right)-\frac{1}{b^{2}} \sum_{n-k+1}^{n} y_{i}^{2}<1\right\}, \quad a, b>0 .
$$

Let $\mathcal{D}_{k}^{a, b}$ denote the identity component of the group of Hamiltonian diffeomorphisms of $\mathbb{R}^{2 n}$ supported away from $V_{k}^{a, b}$ and set

$$
\mathcal{D}_{k}:=\bigcup_{a, b>0} \mathcal{D}_{k}^{a, b}
$$


It follows from Lemma 2.1 above that for any contactomorphism $\phi \in G_{k}$ the smoothed symplectomorphism $S_{\varepsilon} \phi: \mathbb{R}^{2 n} \rightarrow \mathbb{R}^{2 n}$ belongs to $\mathcal{D}_{k}^{a, b}$ if $a$ and $\frac{a}{b}$ are small enough. Moreover, $S_{\varepsilon} \phi$ agrees with $S \phi$ outside $V_{k}^{a^{\prime}, b^{\prime}}$ for any $b^{\prime}>0$ and $a^{\prime}>K_{\phi} \varepsilon$, where $K_{\phi}$ is the constant from Lemma 2.1. Thus, although the smoothing $S_{\varepsilon} \phi$ is not canonical, its action on domains which contain $V_{k}^{a^{\prime}, b^{\prime}}$ with $a^{\prime}>K_{\phi} \varepsilon$ is independent of the choice of the smoothing.

Now we are ready to give the general definition of the domains which form the class $\mathfrak{C}_{k}$.

Definition 2. A connected open domain $V \in \mathbb{R}^{2 n}$ belongs to $\mathfrak{C}_{k}$ if there exist $a_{1}, b_{1}, a_{2}, b_{2}>0$ and a symplectomorphism $\Phi \in \mathcal{D}_{k}$ such that

$$
V_{k}^{a_{1}, b_{1}} \subset V \subset \Phi\left(V_{k}^{a_{2}, b_{2}}\right) .
$$

The group $\mathcal{D}_{k}$, and hence the group $G_{k}$, acts on $\mathfrak{C}_{k}$ by symplectomorphisms.

Lemma 2.2. (i) For $H \in \mathfrak{g}^{+}\left(U_{k}\right)$ we have $V(H) \in \mathfrak{C}_{k}$.

(ii) For $H \in \mathfrak{g}^{+}\left(U_{k}\right), \phi \in G_{k}$ and $\varepsilon$ sufficiently small we have

$$
S_{\varepsilon} \phi(V(H))=V\left(\operatorname{Ad}_{\phi} H\right) .
$$

(iii) If $H, K \in \mathfrak{g}^{+}\left(U_{k}\right)$ satisfy $H \geq K$, then $V(H) \subset V(K)$.

Proof. Claims (ii) and (iii) are straightforward. To prove (i) we first observe that, since the class $\mathfrak{C}_{k}$ is invariant under the action of the group $\mathcal{D}_{k}$, we can replace $H$ by $\operatorname{Ad}_{g}(H)$ for any $g \in G_{k}$. Hence we can assume without loss of generality that $\left.H\right|_{S^{2 n-1} \cap \Pi_{k}^{\perp}}>0$. For $(x, y) \in \mathbb{R}^{2 n}$ we denote

$$
u:=|x|^{2}+\sum_{1}^{n-k} y_{j}^{2}, \quad v:=\sum_{n-k+1}^{n} y_{j}^{2}, \quad r=u+v=|x|^{2}+|y|^{2}, \quad \rho:=\frac{v}{u} .
$$

Then $\rho=\tan ^{2} \alpha$, where $\alpha$ is the angle between the vector $(x, y) \in \mathbb{R}^{2 n}$ and the subspace $\Pi_{k}^{\perp}$. We can view $\rho$ as a function on $S^{2 n-1}$. Take $H \in \mathfrak{g}^{+}$and set $M:=\max (H)$. Then $\left.H\right|_{\rho \geq \rho_{1}}=0$ for a sufficiently large $\rho_{1}$, and for a sufficiently small $\rho_{0}$ we have $m:=\min _{\rho \leq \rho_{0}}(H)>0$. Figure 1 shows that

$$
\partial V(H) \subset \Omega:=\left\{v \leq \rho_{1} u, u+v \geq \frac{1}{M}\right\} \backslash\left\{v<\rho_{0} u, u+v>\frac{1}{m}\right\} .
$$

Consider the hyperboloids

$$
V_{k}^{a, b}=\left\{\frac{u}{a^{2}}-\frac{v}{b^{2}}<1\right\}, \quad V_{k}^{a^{\prime}, b^{\prime}}=\left\{\frac{u}{\left(a^{\prime}\right)^{2}}-\frac{v}{\left(b^{\prime}\right)^{2}}<1\right\} .
$$




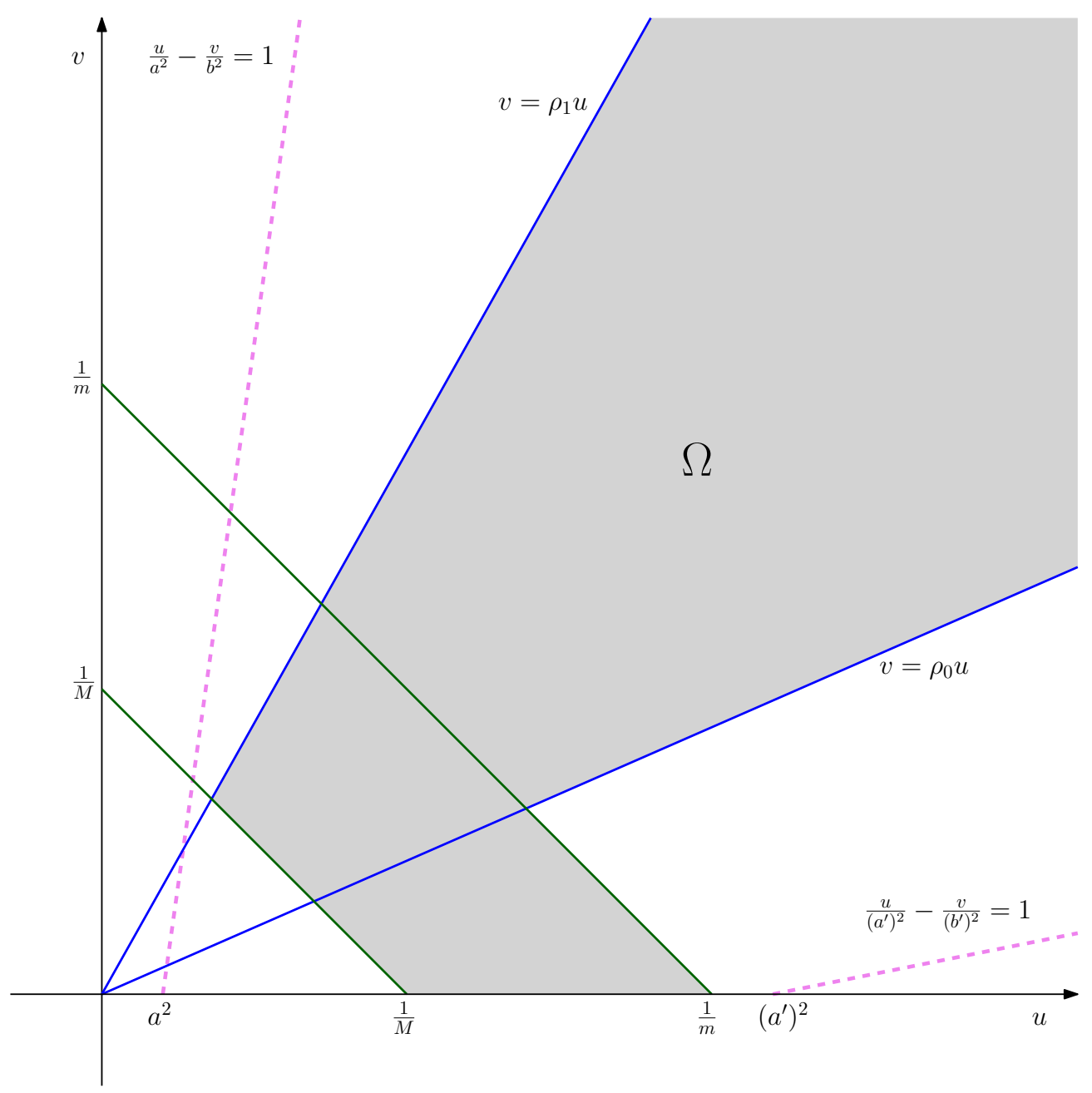

Figure 1: Squeezing $V(H)$ between two hyperboloids

An elementary geometric argument illustrated by Figure 1$]^{1}$ shows that

$$
\Omega \subset V_{k}^{a^{\prime}, b^{\prime}} \backslash V_{k}^{a, b},
$$

provided that

$$
a^{2}<T:=\left(M\left(1+\rho_{1}\right)\right)^{-1}, \quad\left(\frac{b}{a}\right)^{2}>\frac{\rho_{1} T}{T-a^{2}}
$$

and

$$
\left(\frac{b^{\prime}}{a^{\prime}}\right)^{2}<\rho_{0}, \quad\left(a^{\prime}\right)^{2}>\frac{1}{m} .
$$

${ }^{1}$ We thank V. Stojisavljevic for preparing this figure. 
These inequalities express the condition that the dotted lines $\frac{u}{(a)^{2}}-\frac{v}{(b)^{2}}=1$ and $\frac{u}{\left(a^{\prime}\right)^{2}}-\frac{v}{\left(b^{\prime}\right)^{2}}=1$, representing the boundaries of the domains $V_{k}^{a, b}$ and $V_{k}^{a^{\prime}, b^{\prime}}$, do not intersect the shaded region representing $\Omega$. Thus, this choice of $a, b, a^{\prime}, b^{\prime}$ guarantees that $V_{k}^{a, b} \subset V(H) \subset V_{k}^{a^{\prime}, b^{\prime}}$, hence $V(H) \in \mathfrak{C}_{k}$.

\subsection{Capacity-like function on $\mathfrak{C}_{k}$ and proof of Theo- rem 1.11}

The following theorem will be proved in Section 3.4 below.

Theorem 2.3 (Capacity). There exists a capacity-like function $w: \mathfrak{C}_{k} \rightarrow$ $(0, \infty)$ with the following properties:

(i) $w(\Psi(V))=w(V)$ for all $\Psi \in \mathcal{D}_{k}$ and $V \in \mathfrak{C}_{k}$;

(ii) $V \subset V^{\prime}$ implies $w(V) \leq w\left(V^{\prime}\right)$ for all $V, V^{\prime} \in \mathfrak{C}_{k}$;

(iii) $w(s V)=s^{2} w(V)$ for all $s>0$;

(iv) $w\left(V_{k}^{a, b}\right)=\pi a^{2}$ for all $a, b>0$.

Corollary 2.4. For any domain $V \in \mathfrak{C}_{k}$ and $s>1$ there is no $\Psi \in \mathcal{D}_{k}$ such that $\Psi(s V) \subset V$.

Proof. By Theorem 2.3(iii) we have

$$
w(s V)=s^{2} w(V)>w(V),
$$

and the result follows from Theorem 2.3(i) and (ii).

Proof of Theorem 1.11. We define the required function $w: \mathfrak{g}^{+} \rightarrow \mathbb{R}$ by the formula $w(K):=w(V(K))$. For $g \in G_{k}$ we have $V\left(\operatorname{Ad}_{g}(K)\right)=S_{g} \phi(V(K))$, so Theorem 2.3(i) implies that the function $w$ is constant on orbits of the adjoint action. We have $V(s K)=s^{-2} V(K)$, hence $w(s K)=s^{-2} w(K)$ in view of Theorem 2.3(iii). This also yields surjectivity of $w$, and Theorem 2.3(ii) implies that if $w(H)<w(K)$ then there is no contactomorphism $g \in G$ such that $\operatorname{Ad}_{g} H \leq K$. 


\section{Invariants of domains from $\mathfrak{C}_{k}$}

\subsection{Floer-Hofer symplectic homology of bounded do- mains in $\mathbb{R}^{2 n}$}

Filtered symplectic homology $S H^{(a, b)}(U)$ of a bounded open set $U$ in the standard symplectic $\mathbb{R}^{2 n}$ was introduced by A. Floer and H. Hofer in [14] as a far-reaching generalization of earlier symplectic invariants, such as Gromov's symplectic width [17], and later symplectic capacities, see [18]. Since then the invariant has been greatly generalized and expanded, but for the purposes of this paper we will use the original Floer-Hofer version up to the following slight modification. Instead of taking as in [14] a direct limit over Hamiltonians which are negative on $U$ and equal a positive definite quadratic form at infinity, we will take an inverse limit over nonpositive Hamiltonians with compact support in $U$. This version enjoys the same functorial properties as the one in [14] but will be more convenient for the computations below. For domains with smooth boundary of restricted contact type, our version of symplectic homology differs from the one in [14] only by a degree shift of -1 (this follows e.g. from the duality results in [7]). We use $\mathbb{Z} / 2$-coefficients and grade all groups by Conley-Zehnder index.

Let $\mathcal{D}$ denote the group of (not necessarily compactly supported) Hamiltonian diffeomorphisms of $\mathbb{R}^{2 n}$. The following proposition summarizes some relevant properties of symplectic homology, see [14, 15].

Theorem 3.1 (Floer-Hofer). Filtered symplectic homology assigns to each bounded open subset $U \subset \mathbb{R}^{2 n}$ and numbers $0 \leq a<b<\infty$ a $\mathbb{Z}$-graded $\mathbb{Z} / 2$-vector space $S H^{(a, b)}(U)$ with the following properties.

(Functoriality) Each $\Psi \in \mathcal{D}$ induces isomorphisms

$$
\Psi_{*}: S H^{(a, b)}(U) \stackrel{\cong}{\longrightarrow} S H^{(a, b)}(\Psi(U)) .
$$

(Transfer map) Each inclusion $\iota: U \hookrightarrow V$ induces a homomorphism

$$
\iota !: S H^{(a, b)}(V) \rightarrow S H^{(a, b)}(U)
$$

It follows that for $\Psi \in \mathcal{D}$ with $\Psi(U) \subset V$, the inclusion $\iota: \Psi(U) \hookrightarrow V$ together with $\Psi$ induces a homomorphism

$$
\Psi_{!}:=\Psi_{*}^{-1} \circ \iota_{!}: S H^{(a, b)}(V) \rightarrow S H^{(a, b)}(U) .
$$

(Isotopy invariance) For a smooth family $\Psi^{s} \in \mathcal{D}$ with $\Psi^{s}(U) \subset V$ for all $s \in[0,1]$, the maps $\Psi_{!}^{s}: S H^{(a, b)}(V) \rightarrow S H^{(a, b)}(U)$ are independent of $s$. 
(Window increasing homomorphism) For $0 \leq a<b$ and $0 \leq a^{\prime}<b^{\prime}$ with $a \leq a^{\prime}$ and $b \leq b^{\prime}$ we have natural homomorphisms

$$
S H^{(a, b)}(U) \rightarrow S H^{\left(a^{\prime}, b^{\prime}\right)}(U) .
$$

(Symplectic homology of a ball) The symplectic homology in the action window $(0, c)$ of the ball $B_{a}^{2 n}$ of radius a in $\mathbb{R}^{2 n}$ is given by

$$
S H_{j}^{(0, c)}\left(B_{a}^{2 n}\right) \cong \begin{cases}\mathbb{Z} / 2, & c>\pi a^{2} \text { and } j=n, \\ \mathbb{Z} / 2, & c>\pi a^{2} \text { and } j=n\left(2\left\lfloor\frac{c}{\pi a^{2}}\right\rfloor-1\right)-1, \\ 0, & \text { otherwise. }\end{cases}
$$

\subsection{Symplectic homology for domains from $\mathfrak{C}_{k}$}

We extend the definition of symplectic homology to unbounded open domains $V \subset \mathbb{R}^{2 n}$ by

$$
S H^{(a, b)}(V):=\lim _{\longleftarrow} S H^{(a, b)}(U)
$$

where the inverse limit is taken over all bounded open subsets $U \subset V$. We also define symplectic homology in the infinite action window $(a, \infty)$ as

$$
S H^{(a, \infty)}(V):=\lim _{c \rightarrow \infty} S H^{(a, c)}(V) .
$$

The extended symplectic homology still satisfies the properties in Theorem 3.1. However, the invariants one can extract from these general properties are not sufficient for our purposes. Instead, we will concentrate on the special class $\mathfrak{C}_{k}$ of unbounded domains introduced in Section 2 above and study their invariants under the smaller group $\mathcal{D}_{k}$ which preserves this class.

We begin with the computation of symplectic homology of the hyperboloids $V_{k}^{a, b}$.

Proposition 3.2. Let $1 \leq k<n$ and $0<c<\infty$. Then:

(a) For $a, b>0$ we have

$$
S H_{j}^{(0, c)}\left(V_{k}^{a, b}\right) \cong \begin{cases}\mathbb{Z} / 2, & c>\pi a^{2} \text { and } j=n-k, \\ \mathbb{Z} / 2, & c>\pi a^{2} \text { and } j=(n-k)\left(2\left\lfloor\frac{c}{\pi a^{2}}\right\rfloor-1\right)-1, \\ 0, & \text { otherwise. }\end{cases}
$$

(b) For $\widetilde{a}>a, \frac{\widetilde{b}}{\tilde{a}}<\frac{b}{a}$ and $c>\pi \widetilde{a}^{2}$ the transfer map

$$
\mathbb{Z} / 2=S H_{n-k}^{(0, c)}\left(V_{k}^{\widetilde{a}, \widetilde{b}}\right) \rightarrow S H_{n-k}^{(0, c)}\left(V_{k}^{a, b}\right)=\mathbb{Z} / 2
$$


is an isomorphism.

(c) For $c>\pi a^{2}$ the window increasing homomorphism

$$
\mathbb{Z} / 2=S H_{n-k}^{(0, c)}\left(V_{k}^{a, b}\right) \rightarrow S H_{n-k}^{(0, \infty)}\left(V_{k}^{a, b}\right)=\mathbb{Z} / 2
$$

is an isomorphism.

Heuristically, this result is easy to understand: All closed characteristics on the boundary of $V_{k}^{a, b}$ satisfy $z_{n-k+1}=\cdots=z_{n}=0$, so they agree with the closed characteristics on the boundary of the ball $B_{a}^{2(n-k)}$ of radius $a$ in $\mathbb{R}^{2(n-k)}$ whose symplectic homology is given in Theorem 3.1. The actual proof of Proposition 3.2 will be given in Section 3.5 below.

Corollary 3.3. For any domain $V \in \mathfrak{C}_{k}$ there exists $c_{0}$ such that for each $c \geq c_{0}$ and for all $a, b$ with $V_{k}^{a, b} \subset V$, the composition

$$
\alpha_{V, c}: S H_{n-k}^{(0, c)}(V) \rightarrow S H_{n-k}^{(0, c)}\left(V_{k}^{a, b}\right) \rightarrow S H_{n-k}^{(0, \infty)}\left(V_{k}^{a, b}\right)=\mathbb{Z} / 2
$$

(where the first map is the transfer map and the second one the window increasing homomorphism) is surjective.

Proof. Let $V \in \mathfrak{C}_{k}$ and $V_{k}^{a, b} \subset V$. By definition, there exist $a_{1}, b_{1}, a_{2}, b_{2}>0$ and $\Phi \in \mathcal{D}_{k}$ such that $V_{k}^{a_{1}, b_{1}} \subset V \subset \Phi\left(V_{k}^{a_{2}, b_{2}}\right)$. We choose $a_{1}$ so small that

$$
V_{k}^{a_{1}, b_{1}} \subset V_{k}^{a, b} \subset V \subset \Phi\left(V_{k}^{a_{2}, b_{2}}\right)
$$

and $\Phi_{s}=$ id on $V_{k}^{a_{1}, b_{1}}$ for all $s \in[0,1]$, where $\Phi_{s}$ is the isotopy in $\mathcal{D}_{k}$ from the identity to $\Phi_{1}=\Phi$. Set $c_{0}:=\pi a_{2}^{2}$ and consider $c>c_{0}$. Then $\Phi_{s}^{-1}\left(V_{k}^{a_{1}, b_{1}}\right)=V_{k}^{a_{1}, b_{1}} \subset V_{k}^{a_{2}, b_{2}}$, so by isotopy invariance the map

$$
\left(\Phi_{s}^{-1}\right) !: \mathbb{Z} / 2=S H_{n-k}^{(0, c)}\left(V_{k}^{a_{1}, b_{1}}\right) \rightarrow S H_{n-k}^{(0, c)}\left(V_{k}^{a_{2}, b_{2}}\right)=\mathbb{Z} / 2
$$

is independent of $s \in[0,1]$, hence an isomorphism by Proposition 3.2 (b). It follows that the composition of the obvious maps

$$
\begin{aligned}
\mathbb{Z} / 2 & =S H_{n-k}^{(0, c)}\left(V_{k}^{a_{2}, b_{2}}\right) \cong S H_{n-k}^{(0, c)}\left(\Phi\left(V_{k}^{a_{2}, b_{2}}\right)\right) \rightarrow S H_{n-k}^{(0, c)}(V) \\
& \rightarrow S H_{n-k}^{(0, c)}\left(V_{k}^{a, b}\right) \rightarrow S H_{n-k}^{(0, c)}\left(V_{k}^{a_{1}, b_{1}}\right)=\mathbb{Z} / 2
\end{aligned}
$$

is an isomorphism. This implies that $S H_{n-k}^{(0, c)}\left(V_{k}^{a, b}\right)=\mathbb{Z} / 2$ and the map $S H_{n-k}^{(0, c)}(V) \rightarrow S H_{n-k}^{(0, c)}\left(V_{k}^{a, b}\right)$ is surjective, which combined with Proposition 3.2 (c) proves the corollary. 


\subsection{A capacity for domains from $\mathfrak{C}_{k}$}

For any $c>0$ and $V \in \mathfrak{C}_{k}$ we define the augmentation

$$
\alpha_{V, c}: S H_{n-k}^{(0, c)}(V) \rightarrow S H_{n-k}^{(0, \infty)}\left(V_{k}^{a, b}\right)=\mathbb{Z} / 2
$$

as in Corollary 3.3, where $V_{k}^{a, b} \subset V$. For another hyperboloid $V_{k}^{a^{\prime}, b^{\prime}} \subset V$ we find a hyperboloid $V_{k}^{a_{1}, b_{1}} \subset V_{k}^{a, b} \cap V_{k}^{a^{\prime}, b^{\prime}}$, and the commuting diagram

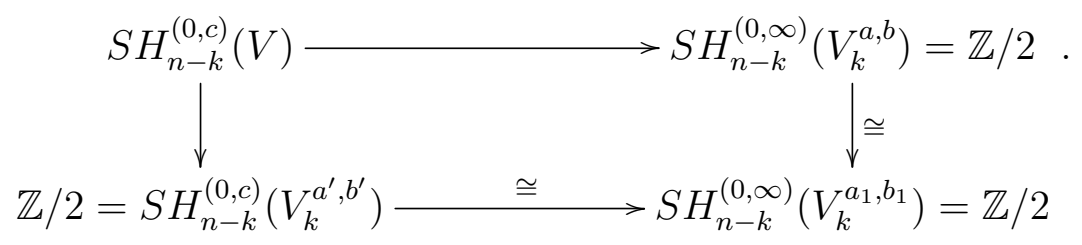

shows that $\alpha_{V, c}$ does not depend on the choice of $a, b$. Corollary 3.3 shows that $a_{V, c}$ is trivial for sufficiently small $c$ and surjective for sufficiently large $c$. The following corollary now is immediate from the properties of symplectic homology in Theorem 3.1 .

Corollary 3.4. (i) For $\Psi \in \mathcal{D}_{k}$ and $a, b$ such that $\Psi=$ id on $V_{k}^{a, b}$ we have the commutative diagram

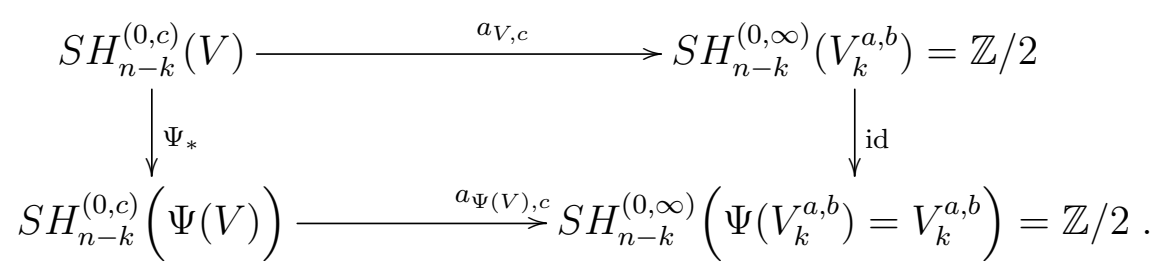

(ii) For an inclusion $\iota: V \hookrightarrow V^{\prime}$ we have the commuting diagram

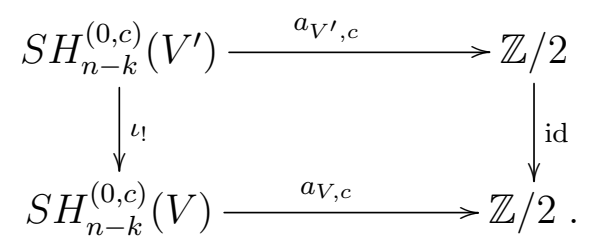

\subsection{Proof of Theorem 2.3}

As in [15], we define the capacity-like function $w: \mathfrak{C}_{k} \rightarrow(0, \infty]$ on $V \in \mathfrak{C}^{k}$ by

$$
w(V):=\inf \left\{c \mid a_{V, c}: S H_{n-k}^{(0, c)}(V) \rightarrow \mathbb{Z} / 2 \text { is surjective }\right\} .
$$


Let us verify that the function $w$ has all the properties in Theorem 2.3. Properties (i) and (ii) follow from the commuting diagrams in Corollary 3.4 , and (iv) follows from Proposition 3.2. Property (iii) follows as in [15], noting that the conformal rescaling $z \mapsto s z$ on $\mathbb{R}^{2 n}$ preserves the class of domains $\mathfrak{C}_{k}$ and multiplies actions by $s^{2}$.

\subsection{Proof of Proposition 3.2}

To compute symplectic homology of the unbounded domain $V=V_{k}^{(a, b)}$, we first need to choose an increasing family $V_{C} \subset V$ of bounded subdomains exhausting $V$. By definition of the inverse limit it is up to us which sequence to choose, and we do it carefully to control the dynamics of the arising Hamiltonian vector fields.

For $C>0$ set $g_{C}(t):=\max \left(-t^{2}, 3 t^{2}-4 C^{2}\right)$ and let $\widetilde{g}_{C}: \mathbb{R} \rightarrow \mathbb{R}$ be a smoothing of the function $g_{C}$. We do the smoothing such that for small $\varepsilon>0$

$$
\widetilde{g}_{C}(t)= \begin{cases}-t^{2}, & |t| \leq C-\varepsilon \\ 3 t^{2}-4 C^{2}, & |t| \geq C+\varepsilon\end{cases}
$$

and $\widetilde{g}_{C}$ has minima at the points $\pm C$ with the minimal value $g_{C}(C)=-C^{2}+$ $\varepsilon$. Moreover, we can choose $\widetilde{g}_{C}$ such that

$$
\frac{t}{2} \widetilde{g}_{C}(t) \geq \widetilde{g}_{C}(t) \quad \text { for all } t \in \mathbb{R} .
$$

Consider the function $H_{C}(x, y):=\frac{x^{2}}{a^{2}}+\frac{\widetilde{g}_{C}(y)}{b^{2}}$ on $\mathbb{R}^{2}$, thus

$$
H_{C}(x, y)= \begin{cases}\frac{x^{2}}{a^{2}}-\frac{y^{2}}{b^{2}}, & |y| \leq C-\varepsilon, \\ -\frac{4 C^{2}}{b^{2}}+\frac{x^{2}}{a^{2}}+\frac{3 y^{2}}{b^{2}}, & |y| \geq C+\varepsilon .\end{cases}
$$

The critical points of $H_{C}$ are a saddle point at the origin and two minima at $(0, \pm C)$ with the minimal value $\frac{C^{2}+\varepsilon}{b^{2}}$. Note that $H_{C}(x, y) \geq \frac{x^{2}}{a^{2}}-\frac{y^{2}}{b^{2}}$. Moreover, condition (2) implies

$$
\frac{1}{2}\left(x \frac{\partial H_{C}}{\partial x}+y \frac{\partial H_{C}}{\partial y}\right)=\frac{x^{2}}{a^{2}}+\frac{y \widetilde{g}_{C}^{\prime}(y)}{2 b^{2}} \geq H_{C}(x, y) .
$$

Lemma 3.5. The Hamiltonian system of $H_{C}$ on the plane $\left(\mathbb{R}^{2}, d x \wedge d y\right)$ has the folowing properties:

(i) all its trajectories except the two homoclinic orbits at the origin are closed; 
(ii) for every closed trajectory $\gamma$ we have $\int_{\gamma} x d y \geq 0$;

(iii) for every nonconstant closed trajectory $\gamma$ with $\left.H_{C}\right|_{\gamma} \geq-\frac{C^{2}}{4 b^{2}}$ we have $\int_{\gamma} x d y \geq C^{2} A_{a, b}$, with a constant $A_{a, b}$ depending only on $a$ and $b$.

Proof. The zero level set of $H_{C}$ forms a figure eight consisting of the two homoclinic orbits at the origin and enclosing the two minima. Assertions (i) and (ii) follow from this picture. For (iii), consider the closed trajectories $\gamma_{ \pm}$ of value $\left.H_{C}\right|_{\gamma_{ \pm}}=-\frac{C^{2}}{4 b^{2}}$ enclosing the points $(0, \pm C)$. Let $V_{ \pm}$be the region bounded by $\gamma_{ \pm}$. Then $V_{ \pm} \cap\{ \pm y \geq C+\varepsilon\}=E \cap\{ \pm y \geq C+\varepsilon\}$ for the ellipse $E=\left\{\frac{x^{2}}{a^{2}}+\frac{3 y^{2}}{b^{2}} \leq \frac{15 C^{2}}{4 b^{2}}\right\}$, and rescaling by $C$ shows that the area of $V_{ \pm} \cap\{ \pm y \geq C+\varepsilon\}$ equals $C^{2} A_{a, b}$ with a constant $A_{a, b}$ depending only on $a$ and $b$. This proves $\int_{\gamma_{ \pm}} x d y \geq C^{2} A_{a, b}$, and the area of each nonconstant closed trajectory $\gamma$ with $\left.H_{C}\right|_{\gamma}>-\frac{C^{2}}{4 b^{2}}$ is larger than this one.

Define now the open domain

$$
V_{C}:=\{G<1\} \subset \mathbb{R}^{2 n}
$$

with the Hamiltonian

$$
G\left(x_{1}, \ldots, x_{n}, y_{1}, \ldots, y_{n}\right):=\sum_{1}^{n-k} \frac{x_{j}^{2}+y_{j}^{2}}{a^{2}}+\sum_{n-k+1}^{n} H_{C}\left(x_{j}, y_{j}\right) .
$$

Lemma 3.6. $V_{C}$ is a bounded domain contained in $V_{k}^{a, b}$ with smooth restricted contact type boundary $\partial V_{C}$. The closed characteristics on $\partial V_{C}$ fall into two groups:

(i) closed characteristics on the sphere $S$ of radius a in the subspace $\mathbb{R}^{2(n-k)} \subset$ $\mathbb{R}^{2 n}$, of actions $k \pi a^{2}$ for $k \in \mathbb{N}$;

(ii) all other closed characteristics have actions $\geq C^{2} B_{a, b}$, with a constact $B_{a, b}$ depending only on $a$ and $b$.

Proof. $V_{C}$ is a bounded because $G$ is exhausting, and $V_{C} \subset V_{k}^{a, b}$ follows from $H_{C}(x, y) \geq \frac{x^{2}}{a^{2}}-\frac{y^{2}}{b^{2}}$. Its boundary is of restricted contact type because it is transverse to the Liouville vector field $Z=\frac{1}{2} \sum_{1}^{n}\left(x_{i} \frac{\partial}{\partial x_{i}}+y_{i} \frac{\partial}{\partial y_{i}}\right)$, which in turn follows from (3) by computation at points of $\partial V_{C}=\{G=1\}$ :

$$
\begin{aligned}
Z \cdot G & =\sum_{1}^{n-k} \frac{x_{j}^{2}+y_{j}^{2}}{a^{2}}+\sum_{n-k+1}^{n} \frac{1}{2}\left(x_{j} \frac{\partial H_{C}}{\partial x_{j}}+y_{j} \frac{\partial H_{C}}{\partial y_{j}}\right) \\
& =1+\sum_{n-k+1}^{n}\left(\frac{1}{2}\left(x_{j} \frac{\partial H_{C}}{\partial x_{j}}+y_{j} \frac{\partial H_{C}}{\partial y_{j}}\right)-H_{C}\left(x_{j}, y_{j}\right)\right) \geq 1
\end{aligned}
$$


Let us study the periodic orbits on $\partial V_{C}$. First, we observe that the corresponding Hamiltonian system with the Hamiltonian $G$ is completely integrable and has integrals

$$
G_{j}(x, y):= \begin{cases}\frac{x_{j}^{2}+y_{j}^{2}}{a^{2}}, & j=1, \ldots, n-k, \\ H_{C}\left(x_{j}, y_{j}\right), & j=n-k+1, \ldots, n .\end{cases}
$$

Hence the simple periodic orbits are given by equations $G_{j}=c_{j}$ for $j=$ $1, \ldots, n$, with $\sum_{1}^{n} c_{j}=1$.

Let us make an accounting of periodic orbits. First of all we have orbits $\left\{x_{n-k+1}=y_{n-k+1}=\ldots x_{n}=y_{n}=0, G_{j}=c_{j} \geq 0, j=1, \ldots, n-k\right\}$ with $\sum_{1}^{n-k} c_{j}=1$ which foliate the sphere $S$ of radius $a$ in the subspace $\mathbb{R}^{2(n-k)} \subset$ $\mathbb{R}^{2 n}$. These orbits and their multiples correspond to group (i) in the lemma and their actions are $k \pi a^{2}$ for $k \in \mathbb{N}$.

Consider now a simple periodic orbit $\gamma$ which is not in group (i). Note that $\gamma$ is a product of periodic orbits $\gamma_{j}$ for the Hamiltonians $G_{j}$, and each $\gamma_{j}$ has nonnegative action by Lemma 3.5(ii). By assumption, at least one of the orbits $\gamma_{j}, j=n-k+1, \ldots, n$, is not the constant orbit at the origin.

If at least one of the constants $c_{j}, j=n-k+1, \ldots, n$ is positive, then the action of the orbit $\gamma_{j}$, and hence of $\gamma$, is $\geq C^{2} A_{a, b}$ by Lemma 3.5(iii). Otherwise, set $\delta:=-\sum_{n-k+1}^{n} c_{j}>0$. Then $\sum_{1}^{n-k} c_{j}=1+\delta$, and therefore $\sum_{1}^{n-k} \int_{\gamma_{j}} x_{j} d y_{j}=\pi(1+\delta) a^{2}$. If $\delta>\frac{C^{2}}{4 b^{2}}$, then the action of the orbit $\gamma$ is $>\pi\left(1+\frac{C^{2}}{4 b^{2}}\right) a^{2}$. Otherwise, all the constants $c_{j}$ for $j=n-k+1, \ldots, n$ satisfy $c_{j} \geq-\frac{C^{2}}{4 b^{2}}$. By assumption, at least one of the corresponding orbits $\gamma_{j}$ is nonconstant, so by Lemma 3.5(iii) the action of this $\gamma_{j}$, and hence of $\gamma$, is $\geq C^{2} A_{a, b}$. This proves Lemma 3.6 .

\section{Deformation to a split Hamiltonian}

We write $G=F_{1}+F_{2}$ with the Hamiltonians

$$
F_{1}:=\sum_{1}^{n-k} G_{j}: \mathbb{R}^{2(n-k)} \rightarrow \mathbb{R}, \quad F_{2}:=\sum_{n-k+1}^{n} G_{j}: \mathbb{R}^{2 k} \rightarrow \mathbb{R} .
$$

Recall that $F_{1}, F_{2}$ have the following properties: 
(i) $F_{1}$ and $F_{2}$ are exhausting with $F_{1} \geq-(n-k) \frac{C^{2}+\varepsilon}{b^{2}}$ and $F_{2} \geq 0$;

(ii) $Z_{i} \cdot F_{i} \geq F_{i}$ for the respective Liouville fields $Z_{i}$;

(iii) all periodic orbits of $F_{i}$ have action $\geq 0$;

(iv) all periods of nonconstant periodic orbits of $F_{i}$ are bounded below by some $\delta>0$;

(v) all second partial derivatives of $F_{i}$ are uniformly bounded;

(vi) the (non-Hamiltonian) action of each $k$-fold covered periodic orbit $z_{2}$ of $F_{2}$ satisfies $\mathcal{A}\left(z_{2}\right)=\pi k a^{2} F_{2}\left(z_{2}\right) \geq \pi a^{2} F_{2}\left(z_{2}\right)$.

Consider a family of Hamiltonians $H_{s}: \mathbb{R}^{2 n} \rightarrow \mathbb{R}, s \in \mathbb{R}$, of the form

$$
H_{s}\left(z_{1}, z_{2}\right)=h_{s}\left(F_{1}\left(z_{1}\right), F_{2}\left(z_{2}\right)\right)
$$

with a smooth family of function $h_{s}: \mathbb{R}^{2} \rightarrow \mathbb{R}$ satisfying the following properties:

(i) $h_{s}$ is locally constant in $s$ outside a compact subset of $\mathbb{R}$;

(ii) outside a compact subset of $\mathbb{R}^{2}$ we have $0<\frac{\partial h_{s}}{\partial F_{1}}<\delta$ or $0<\frac{\partial h_{s}}{\partial F_{2}}<\delta$ (or both);

(iii) all second partial derivatives of the function $\mathbb{R}^{3} \rightarrow \mathbb{R},\left(s, F_{1}, F_{2}\right) \mapsto$ $h_{s}\left(F_{1}, F_{2}\right)$ are uniformly bounded.

Lemma 3.7. For $H_{s}$ as above all 1-periodic orbits are contained in a compact set, and for each $c>0$ the Floer homology $F H^{(0, c)}\left(H_{s}\right)$ is well-defined and independent of $s$.

Proof. For each $s$ the 1-periodic orbits of $H_{s}$ are of the form $z=\left(z_{1}, z_{2}\right)$, where the $z_{i}$ satisfy $\dot{z}_{i}=\frac{\partial h_{s}}{\partial F_{i}} X_{F_{i}}\left(z_{i}\right)$. Hence $F_{1}, F_{2}$ are constant along $z$ and $z_{i}(t)=\gamma_{i}\left(\frac{\partial h_{s}}{\partial F_{i}} t\right)$ for periodic orbits $\gamma_{i}$ of $X_{F_{i}}$ of period $\frac{\partial h_{s}}{\partial F_{i}}$. Therefore, conditions (iv) on $F_{i}$ and (ii) on $h_{s}$ imply that all 1-periodic orbits of $X_{H_{s}}$ are contained in a compact set.

Next, let $u: \mathbb{R} \times S^{1} \rightarrow \mathbb{R}^{2 n}$ be a Floer cylinder connecting 1-periodic orbits. It satisfies

$$
u_{s}+i u_{t}+\nabla H_{s}(u)=0,
$$

where $u_{s}, u_{t}$ denotes the partial derivatives with respect to the coordinates $(s, t) \in \mathbb{R} \times S^{1}$. The bounds on the second derivatives of $F_{i}$ and $h$ yield 
uniform bounds $\left|D^{2} H_{s}\right| \leq A$ on the Hessian of $H_{s}$ and $\left|\nabla \partial_{s} H_{s}(u)\right| \leq B|u|$ on the gradient of the $s$-derivative $\partial_{s} H_{s}$. Using this, a standard computation shows that the function $\rho(s, t):=|u(s, t)|^{2}$ satisfies the estimate

$$
\begin{aligned}
\Delta \rho & =\left|u_{s}\right|^{2}+\left|u_{t}\right|^{2}+\left\langle u, i D^{2} H(u) u_{t}-D^{2} H(u) u_{s}+\nabla \partial_{s} H_{s}(u)\right\rangle \\
& \geq\left|u_{s}\right|^{2}+\left|u_{t}\right|^{2}-A|u|\left(\left|u_{s}\right|+\left|u_{t}\right|\right)-B|u|^{2} \geq-\left(\frac{1}{2} A^{2}+B\right) \rho .
\end{aligned}
$$

By an argument in [3], this estimate implies that Floer cylinders for the $s$-dependent Hamiltonian $H_{s}$ remain in a compact set, hence the Floer homology of $H_{s}$ is well-defined and independent of $s$.

Pick a nondecreasing function $\phi: \mathbb{R} \rightarrow \mathbb{R}$ satisfying $\phi(t)=\delta t / 2$ for $t \geq 0$ and $\phi(t) \equiv-m$ for $t \leq \delta$ with some large constant $m$. For $s \in[0,1]$ consider the Hamiltonian

$$
H_{s}=h_{s}\left(F_{1}, F_{2}\right):=(1-s) \phi\left(F_{1}+F_{2}-1\right)+s \phi\left(F_{1}\right)+s \phi\left(F_{2}-1\right) .
$$

Lemma 3.8. For $\phi$ as above with $m>c$ each 1-periodic orbit $\left(z_{1}, z_{2}\right)$ of $H_{s}$ with action in the interval $(0, c)$ satisfies $z_{1} \equiv 0$, and $z_{2}$ is a 1-periodic orbit of $\phi\left(F_{2}-1\right)$ of action $\pi a^{2} k$ for $k=1, \ldots,\left\lfloor\frac{c}{\pi a^{2}}\right\rfloor$.

Proof. Consider a 1-periodic orbit $z=\left(z_{1}, z_{2}\right)$ of $H_{s}$ with action in the interval $(0, c)$. Its components satisfies the equations

$$
\begin{aligned}
& \dot{z}_{1}=\left((1-s) \phi^{\prime}\left(F_{1}+F_{2}-1\right)+s \phi^{\prime}\left(F_{1}\right)\right) X_{F_{1}}\left(z_{1}\right), \\
& \dot{z}_{2}=\left((1-s) \phi^{\prime}\left(F_{1}+F_{2}-1\right)+s \phi^{\prime}\left(F_{2}-1\right)\right) X_{F_{2}}\left(z_{2}\right) .
\end{aligned}
$$

We distinguish three cases.

Case 1: $z_{1}$ is not constant. Then by Lemma 3.5, for the action to be below $c$, each nonconstant component of $z_{1}$ must have value $H_{C} \leq-\frac{C^{2}}{4 b^{2}}$. Since each constant component has value $\leq 0$ and at least one component is nonconstant, we deduce

$$
F_{1}\left(z_{1}\right) \leq-\frac{C^{2}}{4 b^{2}}
$$

It follows that $\phi\left(F_{1}\right)=-m$ and $\phi^{\prime}\left(F_{1}\right)=0$, so $z_{1}$ satisfies the equation $\dot{z}_{1}=(1-s) \phi^{\prime}\left(F_{1}+F_{2}-1\right) X_{F_{1}}\left(z_{1}\right)$. Since $\dot{z}_{1} \neq 0$, we must have $s<1$ and $F_{1}+F_{2}-1>-\delta$. Together with the preceding displayed equation this yields $F_{2}\left(z_{2}\right)>1-\delta-F_{1}\left(z_{1}\right) \geq 1-\delta+\frac{C^{2}}{4 b^{2}}$, which in view of property (vi) of $F_{2}$ implies $\mathcal{A}\left(a_{2}\right)>\pi a^{2}\left(1-\delta+\frac{C^{2}}{4 b^{2}}\right)>c$. So Case 1 cannot occur. 
Case 2: $z_{1}$ is constant but not all its component are zero. We rule this out by distinguishing several cases.

(i) If $X_{F_{1}}=0$, then each components of $z_{1}$ is a critical point of $H_{C}$, with at least one of them being nonzero. Since $H_{C}(0)=0$ and $H_{C}=$ at the minima we conclude $F_{1}\left(z_{1}\right) \leq-\frac{C^{2}+\varepsilon}{b^{2}} \leq-\frac{C^{2}}{4 b^{2}}$, which is ruled out as in Case 1 .

(ii) If $X_{F_{1}} \neq 0$ and $0<s<1$, then we must have $\phi^{\prime}\left(F_{1}+F_{2}-1\right)=\phi^{\prime}\left(F_{1}\right)=0$, hence $\phi\left(F_{1}+F_{2}-1\right)=\phi\left(F_{1}\right)=-m$. Then $z_{2}$ satisfies $\dot{z}_{2}=s \phi^{\prime}\left(F_{2}-1\right) X_{F_{2}}\left(z_{2}\right)$, so by the choice of $\phi$ it can only be 1 -periodic if $\phi\left(F_{2}-1\right) \leq 0$. Thus $H_{s}(z) \leq-m$ and the Hamiltonian action of $z$ satisfies $\mathcal{A}_{H_{s}}(z) \geq m>c$.

(iii) If $X_{F_{1}} \neq 0$ and $s=0$, then $\phi^{\prime}\left(F_{1}+F_{2}-1\right)=0$, hence $H_{0}(z)=$ $\phi\left(F_{1}+F_{2}-1\right)=-m$ and again $\mathcal{A}_{H_{0}}(z) \geq m>c$.

(iv) If $X_{F_{1}} \neq 0$ and $s=1$, then $\phi^{\prime}\left(F_{1}\right)=0$, hence $\phi\left(F_{1}\right)=-m$. Then $\dot{z}_{2}=\phi^{\prime}\left(F_{2}-1\right) X_{F_{2}}\left(z_{2}\right)$ implies $\phi\left(F_{2}-1\right) \leq 0$, thus $H_{1}(z) \leq-m$ and again $\mathcal{A}_{H_{1}}(z) \geq m>c$.

Case 3: $z_{1} \equiv 0$. Then $F_{1}\left(z_{1}\right)=0$ and $z_{2}$ is of the form described in the lemma.

Proof of Proposition 3.2. Lemma 3.7 and Lemma 3.8 together imply (after replacing $H_{s}$ by $H_{\sigma(s)}$ for a nondecreasing function $\sigma: \mathbb{R} \rightarrow[0,1]$ which equals 0 for $s \leq 0$ and 1 for $s \geq 1$ ) that the Floer homology $F H^{(0, c)}\left(H_{s}\right)$ is independent of $s \in[0,1]$. By definition, the Hamiltonian $H_{0}(z)=\phi(G(z)-1)$ computes the symplectic homology of $V_{C}$,

$$
F H^{(0, c)}\left(H_{0}\right) \cong S H^{(0, c)}\left(V_{C}\right) .
$$

The Hamiltonian

$$
H_{1}\left(z_{1}, z_{2}\right)=\phi\left(F_{1}\left(z_{1}\right)\right)+\phi\left(F_{2}\left(z_{2}\right)-1\right)
$$

is split, as well as the corresponding Floer equation. It follows that all its Floer cylinders are contained in the subspace $\mathbb{R}^{2(n-k)}$, so $H_{1}$ computes the symplectic homology of the ball $B_{a}^{2(n-k)}$ of radius $a$ in $\mathbb{R}^{2(n-k)}$. This symplectic homology is computed in [15], up to an index shift by 1 due to our different conventions, to be

$$
S H_{j}^{(0, c)}\left(B_{a}^{2(n-k)}\right) \cong \begin{cases}\mathbb{Z}_{2}, & c>\pi a^{2} \text { and } j=n-k, \\ \mathbb{Z}_{2}, & c>\pi a^{2} \text { and } j=(n-k)\left(2\left\lfloor\frac{c}{\pi a^{2}}\right\rfloor-1\right)-1, \\ 0, & \text { otherwise. }\end{cases}
$$

This proves part (a) of Proposition 3.2. Parts (b) and (c) follow by similar arguments. 


\section{Geometry of the dominating positive cone}

Let $\mathfrak{g}^{+}$be the maximal dominating cone of an open contact manifold $(U, \xi)$. The group $\mathbb{R}_{+}$acts on $\Theta:=\mathfrak{g}^{+} / \sim$ by multiplication. We assume that $\mathfrak{g}^{+}$is orderable up to conjugation and consider the binary relation $\preceq$ on $\Theta$ from Section 1.5. For a pair of classes $f, h \in \Theta$ define

$$
\rho(f, h):=\inf \{s>0 \mid f \preceq s h\} .
$$

The fact that $\mathfrak{g}^{+}$is dominating implies that the set on the right hand side is nonempty, and orderability up to conjugation means that there exist $a, b \in \Theta$ such that $\rho(a, b) \neq 0$. Furthermore, clearly we have sub-multiplicativity

$$
\rho(f, h) \leq \rho(f, g) \rho(g, h) \text { for all } f, g, h \in \Theta .
$$

Observe that $\rho(h, h) \geq 1$ for all $h \in \Theta$ by Lemma 1.7 (a). On the other hand, obviously $\rho(h, h) \leq 1$ and hence $\rho(h, h)=1$.

We claim that $\rho(f, g) \neq 0$ for all $f, g \in \Theta$. Indeed, take $a, b$ with $\rho(a, b) \neq 0$ and write, by sub-multiplicativity,

$$
0<\rho(a, b) \leq \rho(a, f) \rho(f, g) \rho(g, b),
$$

yielding $\rho(f, g) \neq 0$. The claim follows.

Define now a function $d: \Theta \times \Theta \rightarrow \mathbb{R}$ by

$$
d(g, h)=\max (|\log \rho(g, h)|,|\log \rho(h, g)|) .
$$

The above discussion shows that $d$ is a pseudo-metric on $\Theta$ : it is symmetric, nonnegative and satisfies the triangle inequality. It is unknown whether $d$ is a genuine distance on $\Theta$ (and sounds unlikely that it is). Introduce the equivalence relation $\approx$ on $\Theta$ by $f \approx g$ whenever $d(f, g)=0$. This relation measures the deviation of $d$ from a genuine metric. Interestingly enough, it also measures the deviation of the binary relation $\preceq$ from a genuine partial order. Indeed, if $f \preceq g$ and $g \preceq f$, then we have $\rho(\bar{f}, g) \leq 1$ and $\rho(g, f) \leq 1$. By (5),

$$
1=\rho(f, f) \leq \rho(f, g) \rho(g, f),
$$

and hence

$$
\rho(f, g)=\rho(g, f)=1 .
$$

This yields $d(f, g)=0$ and hence $f \approx g$. Denote $\Xi:=\Theta / \approx$, and note that the pseudo-metric $d$ descends to $\Xi$ as a genuine metric $D$. What about the partial order? Define a relation $\ll$ on $\Xi$ as follows: $p \ll q$ if there exist 
$f, g \in \Theta$ and a sequence of positive numbers $\varepsilon_{i} \rightarrow 0$ so that $p=[f], q=[g]$ and $f \preceq\left(1+\varepsilon_{i}\right) g$ for all $i$. (The $\varepsilon_{i}>0$ are needed because of the infimum in (4q)). Note that this is a transitive and reflexive relation. We claim that $\ll$ is a genuine partial order. Indeed if $p \ll q$ and $q \ll p$, then $D(p, q)=0$ and hence $p=q$. It would be interesting to explore the geometry of $\Xi$ with respect to $D$.

Remark 4.1. The quantity $\rho$ and the pseudo-metric $d$ has cousins in the earlier literature. On the one hand, they can be considered Lie algebra counterparts of the relative growth between positive contactomorphisms, and the corresponding pseudo-metric, respectively, studied in [13]. On the other hand, each compactly supported non-negative function $H$ on $U$ defines a "contact form" $\alpha / H$ on $U$, where the quotation marks stand for the fact that this form could be infinite and certainly is infinite outside a compact set. With this language, the adjoint action of contactomorphisms on functions corresponds to the action of contactomorphisms on contact forms, and the metric $d$ is an analogue, for open manifolds, of the contact Banach-Mazur distance introduced by Yaron Ostrover and the third-named author and discussed in [19, 20] in the context of closed contact manifolds.

Example 4.2. Consider $\left(S^{2 n-1} \backslash \Pi_{k}, \xi_{s t}\right)$ as above with $k<n$. The function $w: \mathfrak{g}^{+} \rightarrow(0, \infty)$ from Theorem 1.11 descends to a function $\widetilde{w}: \Theta \rightarrow(0, \infty)$ with the following properties:

(i) $h \preceq f \Rightarrow \widetilde{w}(h) \geq \widetilde{w}(f)$;

(ii) $\widetilde{w}(s h)=s^{-2} \widetilde{w}(h)$ for all $s>0, h \in \Theta$.

These properties readily yield the following inequality:

$$
d(f, h) \geq \frac{1}{2}\left|\log \frac{\widetilde{w}(f)}{\widetilde{w}(h)}\right| .
$$

For instance, this shows that $d(g, s g)=|\log s|$, and in particular the restriction of the pseudo-metric $d$ to each orbit of the $\mathbb{R}_{+}$-action on $\Theta$ is isometric to the Euclidean line. It would be interesting to explore whether $\Theta$ admits a quasi-isometric embedding of the Euclidean $\mathbb{R}^{N}$ for $N \geq 2$. Let us mention also that the above conclusions continue to hold verbatim for the metric space $(\Xi, D)$. 


\section{References}

[1] M.S. Borman, Y. Eliashberg and E. Murphy, Existence and classification of overtwisted contact structures in all dimensions, Acta Math. (2) 215(2015), 281-361.

[2] M. Bhupal, A partial order on the group of contactomorphisms of $\mathbb{R}^{2 n+1}$ via generating functions, Turkish J. Math. 25 (2001), 125-135.

[3] K. Cieliebak, Pseudo-holomorphic curves and periodic orbits on cotangent bundles, J. Math. Pures Appl. (9) 73 (1994), no. 3, 251-278.

[4] K. Cieliebak and Y. Eliashberg, From Stein to Weinstein and Back. Symplectic geometry of affine complex manifolds, American Mathematical Society Colloquium Publications 59 (2012).

[5] K. Cieliebak, Y. Eliashberg and L. Polterovich, in preparation.

[6] K. Cieliebak, A. Floer, H. Hofer and K. Wysocki, Applications of symplectic homology II: Stability of the action spectrum, Math. Z. 223 (1996), no. 1, 27-45.

[7] K. Cieliebak and A. Oancea, Symplectic homology and the EilenbergSteenrod axioms, arXiv:1511.00485.

[8] Y. Eliashberg, New invariants of open symplectic and contact manifolds, J. Amer. Math. Soc. 4 (1991), no. 3, 513-520.

[9] Y. Eliashberg, Contact 3-manifolds twenty years since J. Martinet's work, Ann. Inst. Fourier (Grenoble) 42 (1992), no. 1-2, 165-192.

[10] Y. Eliashberg, Weinstein manifolds revisited, arXiv:1707.03442.

[11] Y. Eliashberg and M. Gromov, Convex symplectic manifolds, Several complex variables and complex geometry, Part 2 (Santa Cruz, CA, 1989), 135-162, Proc. Sympos. Pure Math., 52, Part 2, Amer. Math. Soc., Providence, RI, 1991.

[12] Y. Eliashberg, S. Kim and L. Polterovich, Geometry of contact transformations and domains: orderability versus squeezing, Geom. Topol. 10 (2006), 1635-1747.

[13] Y. Eliashberg and L. Polterovich, Partially ordered groups and geometry of contact transformations Geom. Funct. Anal. 10 (2000), no. 6, 14481476 . 
[14] A. Floer and H. Hofer, Symplectic homology I: Open sets in $\mathbb{C}^{n}$, Math. Z. 215 (1994), no. 1, 37-88.

[15] A. Floer, H. Hofer and K. Wysocki, Applications of symplectic homology I, Math. Z. 217 (1994), no. 4, 577-606.

[16] E. Giroux, Convexité en topologie de contact (French) Comment. Math. Helv. 66 (1991), no. 4, 637-677.

[17] M. Gromov, Pseudo holomorphic curves in symplectic manifolds, Invent. Math. 82 (1985), no. 2, 307-347.

[18] H. Hofer and E. Zehnder, Symplectic invariants and Hamiltonian dynamics, Birkhäuser (1994).

[19] L. Polterovich and J. Zhang, in preparation.

[20] V. Stojisavljević and J. Zhang, in preparation.

[21] C. Viterbo, Functors and computations in Floer homology with applications I, Geom. Funct. Anal. 9 (1999), no. 5, 985-1033. 